\title{
Effects of motion on blur discrimination
}

\author{
Ari K. Pääkkönen* and Michael J. Morgan \\ Laboratory for Neuroscience, Department of Pharmacology, University of Edinburgh, 1 George Square, \\ Edinburgh EH8 9JZ, Scotland
}

Received March 1, 1993; revised manuscript received August 17, 1993; accepted August 17, 1993

\begin{abstract}
Blur-discrimination thresholds for moving, Gaussian-blurred edges were measured. Our results show that motion produces equivalent spatial blur. The velocity dependence of this blur is linear, and its extent can be predicted by a temporal impulse response with a standard deviation of $\sim 5 \mathrm{~ms}$ in normal room light. This blur is most likely to result from cameralike summation and not from the use of larger spatial filters for moving than for stationary objects. As a function of reference blur, blur-discrimination thresholds approach asymptotically a Weber's-law relation. Motion-deblurring models are discussed in view of our findings, and an alternative model for temporal integration is proposed.
\end{abstract}

\section{INTRODUCTION}

There are several pattern-discrimination tasks in which the psychophysical discrimination thresholds are smaller than the minimum cone separation. It is rather surprising that some of these hyperacuities, for instance vernier acuity ${ }^{1}$ and stereoscopic acuity, ${ }^{2}$ are unaffected by motion up to velocities of several degrees of arc per second. One would expect from the poor temporal resolution of the visual system - the temporal integration period for both stationary ${ }^{3}$ and moving ${ }^{4,5}$ objects has been estimated to be more than $100 \mathrm{~ms}$ in daylight-that motion blur should degrade the thresholds. The fact that this does not happen has given rise to diverse explanations.

Explanations for the absence of motion degradation in pattern-discrimination tasks have consisted of two main approaches. First, it has been suggested ${ }^{5,6}$ that the visual system has special motion-deblurring or blur-prevention mechanisms to aid in the analysis of the shape of moving objects. In principle, these mechanisms restore positional acuity by taking into account the temporal delay at which different photoreceptors have been stimulated. The second approach ${ }^{7,8}$ does not invoke a general motiondeblurring mechanism. It assumes that image motion does introduce spatial blur. However, motion blur remains undetected because the internal representation of the object is already degraded by spatial blur to such a degree that motion blur does not degrade the information noticeably further.

Burr et al. ${ }^{5}$ used a masking technique to measure the joint spatiotemporal tuning functions of motion detectors, and, assuming linearity at threshold, they calculated the spatiotemporal receptive fields of the detectors by inverse Fourier transform of the data. The fields obtained comprise alternating ridges of opposite polarity, elongated in space-time along the preferred velocity axis of the detector. Burr et al. used the concept of a spatiotemporal receptive field to explain many phenomena of motion perception, one of which is the blur-free perception of moving objects. The essence of the explanation is that a spatiotemporally oriented receptive field integrates a moving object not statically but along its path of motion. If the orientation of the receptive field corresponds exactly to the velocity of the object, integration produces no smear.

Anderson and van Essen ${ }^{6}$ argued that in many computational visual problems it is desirable to control dynamically how the retinal output maps onto higher levels. One of their examples was the prevention of blurring of moving images. They proposed a general computational strategy for resolving these problems. Their solution, which they termed shifter circuits, involves dynamic shifts in the relative alignment of input and output maps without loss of local spatial relationships. The shifts are produced in increments along a succession of relay stages connected by ascending, symmetrically diverging excitatory connections. At each stage the shifts are controlled by lateral inhibitory connections that selectively suppress appropriate sets of ascending inputs. A descending visually driven feedback loop is needed to give a measure of the alignment to the shift control circuitry. In the case of motion-blur prevention, the feedback signals the locally measured retinal velocity of the object. The basic principle of both the shifter hypothesis and the spatiotemporal receptive-field hypothesis is the same: integration follows the path of motion of the object. The implementation, however, is different: spatiotemporal receptive fields are stationary and are made up of hard-wired connections, whereas shifter circuits use dynamic switches and in fact shift whole receptive fields.

Morgan and Benton ${ }^{7}$ confirmed the finding of Westheimer and $\mathrm{McKee}^{1}$ by showing that observers can detect a vernier cue of less than 10 arcsec and that acuity is little affected by target motions up to $6 \mathrm{deg} / \mathrm{s}$. But they also found that spatial-interval discimination thresholds for a bar separation of 4.5 arcmin rise approximately threefold over the velocity range of $0-6 \mathrm{deg} / \mathrm{s}$. They took these different motion sensitivities of different hyperacuity tasks as evidence against general motion-deblurring mechanisms. According to their explanation, spatial-interval acuity is more sensitive to motion blur than is vernier acuity, for the following reason. In the two-bar-interval case, the bars follow one another rapidly at the same retinal location. Motion blur, or, in other words, the sluggish temporal resolution of the visual system, causes the bars' 
responses to overlap, and their peaks become difficult to discriminate. Morgan and Benton predicted from this that spatial-interval acuity would be less affected by motion if the separation between the bars were increased, and their results confirmed this prediction. On the other hand, however blurred the vertical vernier lines are by horizontal motion, they do not overlap on the retina. Morgan and Benton concluded that the insensitivity of vernier acuity to motion depends not on the removal of motion blur but on the fact that blur (either static or motion induced) does not degrade the critical information in the stimulus.

Morgan and Benton's explanation for different motion sensitivites of the two hyperacuity tasks leaves some questions. Let us assume, for instance, that there is a motion-deblurring mechanism that removes most of (but not all) the motion blur predicted from the temporal summation period of the visual system. A mechanism removing all the motion blur would not be biologically plausible: no neural mechanism can have exact temporal accuracy. Let us assume further that the residual motion blur is treated as an equivalent of a small amount of spatial blur in the later stages of analysis. If we then have two hyperacuity tasks that have different sensitivities to spatial blur, it is easy to see that the tasks will also have different sensitivities to motion. Thus having different motion sensitivities for different hyperacuity tasks is not a sufficient proof against a general motion-deblurring mechanism if the sensitivities of these tasks to spatial blur are not equal. To study the issue properly one needs psychophysical tasks in which the effects of static spatial blur and motion blur can be separated on a quantitative basis.

To compare the effects of motion blur and static spatial blur, in this paper we examine blur discrimination for moving, Gaussian-blurred edges. Our psychophysical experiment is a motion-domain extension of work by Watt and Morgan, ${ }^{9}$ who measured blur-discrimination thresholds for stationary edges. They found that thresholds depend on the reference blur according to a U-shaped function: thresholds decrease as the reference blur is increased from zero to an optimum level, beyond which thresholds rise corresponding approximately to a power law with an exponent of 1.5. In this study we measured blur-discrimination thresholds as a function of both reference blur and velocity. Our prediction was that an increase in stimulus velocity should increase the internal blur and shift the thresholds accordingly.

\section{GENERAL METHODS}

Data were collected from one of the authors (AP) and two other observers (RO and $\mathrm{MS}$ ) who were naïve regarding the purpose of the study. Observers RO and MS had normal uncorrected vision. Observer AP had uncorrected acuity of approximately half of the normal mean value but no refractive errors. Subjects viewed the display binocularly with natural pupils $100 \mathrm{~cm}$ from the screen. Normal room lighting provided a background illumination of $25 \mathrm{~cd} / \mathrm{m}^{2}$, ensuring that all observations were carried out at photopic levels.

Figure 1 illustrates the stimulus arrangement. Stimuli were displayed on a Hewlett-Packard Model 1333a oscilloscope with a brief-persistence P15 phosphor. The spot size of this oscilloscope is less than $0.3 \mathrm{~mm}$, and the brightness of an intensified spot diminishes to $0.1 \%$ of its original value in less than $50 \mu \mathrm{s}$. The stimuli were generated by a Macintosh IIcx computer that also carried out most of the other input, decision-making, and output tasks necessary for the execution of the experiment. Digital $x$ and $y$ position information for each dot were converted to analog voltage levels by a digital-to-analogconverter card. A 140-arcmin-long horizontal line was produced by generation of a row of unresolved dots placed 0.3 arcmin apart. A $400-\mathrm{kHz}$ triangle wave signal generated by a function generator was then added to the $y$ signal to transform the horizontal line to a horizontal band 20 arcmin wide. We moved the band by shifting its position less than 2.4 arcmin in every $5 \mathrm{~ms}$ so that the motion appeared to be continuous. The timing of movement and stimulus presentation was controlled by the computer with an accuracy of $\sim 5 \mu \mathrm{s}$. The minimum refresh rate was $400 \mathrm{~Hz}$.

Every band presented comprised a vertical edge of specific blur. The profile of the blurred edge had the form of an integrated Gaussian. Its luminance profile was controlled by a $z$ modulation voltage provided by a third digitalto-analog converter channel. Luminance values of the display were calibrated against the input $z$ voltages by a microphotometer. The minimum luminance of the band was always $28.5 \mathrm{~cd} / \mathrm{m}^{2}$ and the maximum $58.9 \mathrm{~cd} / \mathrm{m}^{2}$, making a Michelson contrast of $35 \%$. The blur width of an edge was specified by the standard deviation of the Gaussian.

On each trial the sequence of the patterns on the oscilloscope screen was the following: the fixation mark, a blank period, the first band, a blank period, the second band. The fixation mark was displayed for $500 \mathrm{~ms}$. To minimize the effects of anticipatory eye movements, we randomized the durations of the blank intervals, the first duration in the range of 300-500 $\mathrm{ms}$ and the second in the range of 450-750 ms. The bands either were stationary or were moved horizontally with identical speed leftward or rightward, direction being randomized for each band. The polarity of the edge was selected so that the lower luminance end of the band was always leading. To minimize the effects of pursuit eye movements, we limited the duration of the presentation of each band to $150 \mathrm{~ms}$.

On a flat screen there is variation in the perceived angular velocity along the motion trajectory, even for an object in a uniform, rectilinear motion. There is also variation in the spatial-filtering properties because of changes in eccentricity. To minimize these effects, especially to avoid changes in the static spatial-filter sizes along the trajectory, we could not let the motion trajectory

\section{$140^{\prime}$}

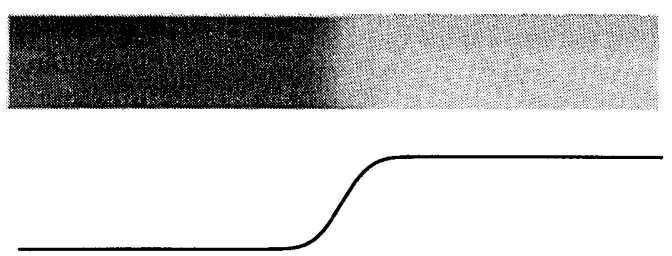

$20^{\prime}$

Fig. 1. Shaded band, stimulus arrangement used in the experiments; line curve, profile of an integrated Gaussian of the luminous vertical edge in the band. 

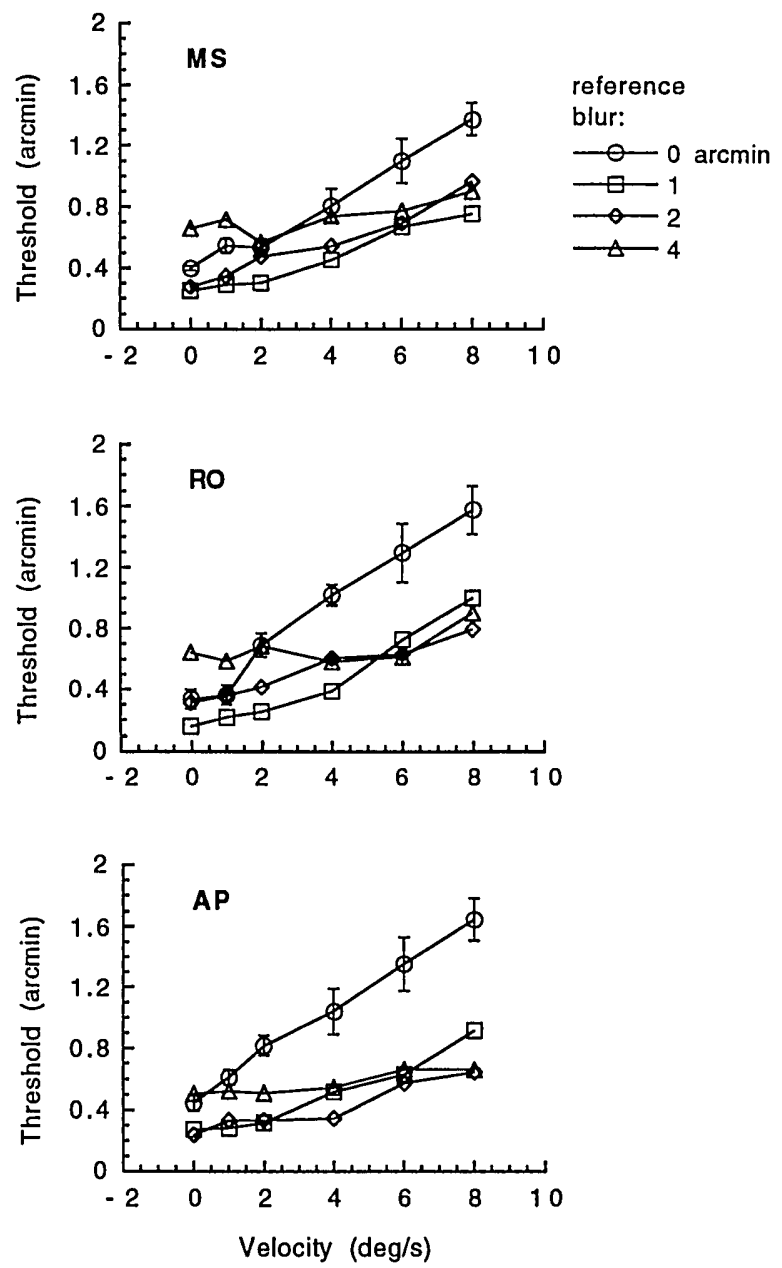

Fig. 2. Blur-discrimination thresholds as a function of velocity at four different reference blurs (space constants $0,1,2$, and 4 arcmin) for observers $\mathrm{MS}$, RO, and AP. The reference-blur space constant is specified by the standard deviation of the Gaussian. The error bars for the zero-arcmin reference blur are presented as an example; they represent \pm 1 standard error. Typically 1 standard error was $\sim 10 \%$ of the threshold.

extend far from the central fovea. Therefore the maximum velocity used in this experiment was limited to $8 \mathrm{deg} / \mathrm{s}$.

One of the bands in each trial always had an edge with the reference-blur width. The reference-blur value was jittered from trial to trial in the range of the nominal reference blur $\pm 10 \%$. Over a series of 64 trials we used an adaptive probit estimation algorithm ${ }^{10}$ to select the cue, i.e., to select the difference between the reference and the test blur randomly from a number of preset magnitudes. The absolute value of this difference was always added to the reference-blur value to produce the test-blur width. The sign of the difference was used to specify whether the band with the reference blur or with a test blur was presented first. We varied the location of the edge within the band randomly in a region of uncertainty 2 arcmin wide to make it impossible for the subject to use distance cues in the measurement of blur. Two series of 64 trials corresponding to situations in which the bands moved either in the same direction or in the opposite directions were randomly interleaved. The analysis of the resultant two psychometric functions was done separately.
The observer's task was to decide whether the edge in the first or in the second band was more blurred. Threshold was defined as the standard deviation of the resultant psychometric function (83\%-correct point), and we estimated it by fitting a cumulative normal curve to the psychometric function, using probit analysis. ${ }^{11}$ Probit analysis also provides the standard error of the estimate for the standard deviation and a chi-square value that can be used in assessing the goodness of fit. At least four thresholds were determined under each condition. Each final value reported represents the root mean square of these estimates. Thresholds for all possible combinations of four different reference blurs $(0,1,2$, and 4 arcmin) and six different velocities $(0,1,2,4,6$, and $8 \mathrm{deg} / \mathrm{s})$ were measured.

\section{RESULTS}

Figure 2 shows the thresholds as a function of velocity with four different reference blurs for all the observers. Although the data of observer AP differ in some aspects from the data of the others, the main features are similar. For each reference blur the discrimination thresholds increase with velocity approximately linearly, and the slope of this increase is inversely related to the reference blur. The smaller the reference blur, the larger the effect of velocity on the thresholds. Blur comparison is at its best not at zero blur but at some higher reference-blur value for all velocities. This is illustrated in Fig. 3, where the thresholds for observer RO are plotted as a function of reference blur. This finding confirms the finding of Watt and Morgan ${ }^{9}$ for stationary Gaussian blur. This optimum blur also seems to shift to higher blur values with velocity. Observer AP's performance is better than that of the other observers at a reference blur of 4 arcmin, whereas the others perform better than he does at smaller reference blurs.

\section{MODEL OF BLUR DISCRIMINATION OF MOVING TARGETS}

The results show that image motion shifts the discrimination thresholds, indicating that motion produces equivalent spatial blur. To estimate the amount of this equivalent blur, we need a model to separate the effects of motion blur and static spatial blur. The use of a mathematical model has a prerequisite: we must assume that the blurdiscrimination system is linear near threshold. Another fact of signal analysis helps us in building the model: in

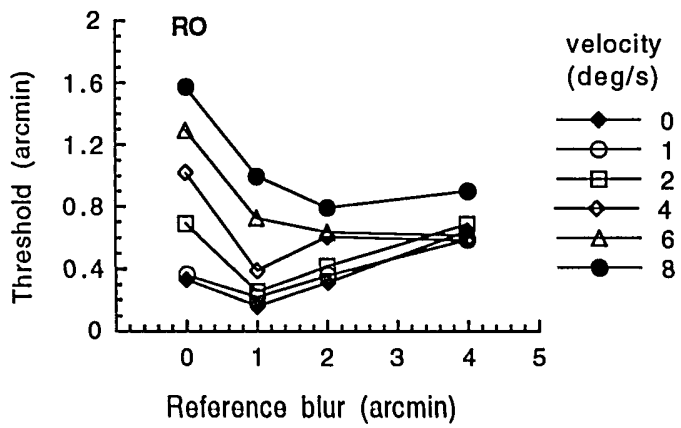

Fig. 3. Blur-discrimination data for observer RO from Fig. 2 replotted as a function of reference blur for six different velocities. The optimum blur is not at zero but at some higher reference-blur value for all velocities. 
mathematical terms blurring is equivalent to filtering, since both are produced by convolution of the signal with a function that can be termed either a blurring function or a spatial filter. In filtering terminology our results indicate an increase in the effective spatial-filter size with velocity.

The spatial filtering of moving stimuli must contain at least some of the same static spatial-filtering operations that blur stationary images, since there is no difference between the optical blurring of stationary and of moving objects. Nor does the mosaic of photoreceptors change in response to moving images. We assume that all the operations that are not velocity dependent are actually the same, and we represent the compound action of these operations with a single static spatial filter of space constant $s$. In addition, our model has a blur component that is velocity dependent. In the simplest case the space constant of this component is the product of a parameter $f$ and a velocity $v$. The choice of method for combining the static and the velocity-dependent spatial filters, as well as the interpretation of the parameter $f$ and the product $f v$, depends on the assumed motion blur type. To clarify this, we give two simplified examples of different motion blur types.

If motion blur is purely cameralike, the filtering can be described in two phases. First, the motion of the object and the temporal response of the photoreceptors together produce the velocity-dependent spatial filtering, and the outputs of the receptors are then combined with the static spatial filter. Mathematically the operation contains two separate, consecutive convolutions, and the total output is separable in terms of static and velocity-dependent spatial filtering. Since the variances are additive in convolution, the space constant of the resultant spatial filter $s_{r}$ is the square root of the sum of the squared space constants of the components:

$$
s_{r}=\left[s^{2}+(f v)^{2}\right]^{1 / 2} .
$$

In the case of cameralike blur, the parameter $f$ is a measure of the exposure or summation time; in fact, it is the standard deviation of the temporal weighting function, or the temporal impulse response of the system.

Another type of motion blurring results if we assume that the visual system uses different spatial filters at different velocities and that the size of these spatial filters increases with velocity. If, for the sake of simplicity of this example, we ignore the temporal response of the photoreceptors, then the filtering operation in this case contains only one convolution. The total output is inseparable in terms of static and velocity-dependent spatial filtering, and the space constant of the filter is simply the sum of the components:

$$
s_{r}=\left[(s+f v)^{2}\right]^{1 / 2}=s+f v .
$$

The velocity-dependent component $f v$ is not a space constant of any realizable filter: it merely indicates how the size of the spatial filter depends on the velocity of the object. The parameter $s$ gives the space constant of the filter for stationary objects.

In reality, the blurring of moving objects is likely to be a rather complex mixture of separable and inseparable blur components. There is no way to avoid the optical blurring that produces in principle a static filtering stage. Furthermore, it is hard to believe that the sluggish temporal response of photoreceptors would not produce any motion blur on moving objects. The static and the velocitydependent blurs produced at these two stages are separable. On the other hand, at the bipolar or ganglion cell level, the temporal filtering in the center of a receptive field is different from the temporal filtering of the surround. ${ }^{12-15}$ Thus the static and the velocity-dependent blurs produced by the center-surround organization are inseparable. There may also be some higher filtering stages producing blur of either or both types. In spite of all this complexity, we can, however, model our data by. using only one static and one velocity-dependent spatialfiltering component. The estimates obtained in this way are the total effective space constants of the components. If we calculate the estimates for two extreme cases, fully separable and fully inseparable blur, we get the lower and upper limits for the effective values.

Our model derivation follows some of the reasoning presented by Watt ${ }^{16}$ for the discrimination of stationary blur. The internal representation of an edge is a blurred image of the real physical appearance of that edge. In the case of fully separable blur, we assume that the representation of the edge is blurred by the two spatial filters: a static one and a velocity-dependent one. In mathematical terms, the representation of the blurred edge is determined by the convolution of the real edge of blur $B$, a static spatial filter with space constant $s$, and a velocitydependent spatial filter with space constant $f v$. The standard deviation or the blur width of the internal representation of the edge $B^{\prime}$ is the square root of the sum of variances of its convolution components:

$$
B^{\prime}=\left[B^{2}+s^{2}+(f v)^{2}\right]^{1 / 2}
$$

We then suppose that there is only one dominant source of error in the judgment of blur difference. This source is the comparison of measurements of internal representations of blurred edges, which introduces a Weber's-law error:

$$
\Delta B^{\prime}=k B^{\prime}
$$

where $k$ is the Weber fraction and $\Delta B^{\prime}$ the threshold in the comparison of internal representations of edges. If we then assume that the internal difference $\Delta B^{\prime}$ is caused by the increment $\Delta B$ to the reference blur, we have

$$
B^{\prime}+\Delta B^{\prime}=\left[(B+\Delta B)^{2}+s^{2}+(f v)^{2}\right]^{1 / 2} .
$$

Substituting first Eq. (4) and then Eq. (2) into Eq. (5) leads to a quadratic equation that can be solved for $\Delta B$ :

$$
\Delta B=-B+\left\{B^{2}+\left(k^{2}+2 k\right)\left[B^{2}+s^{2}+(f v)^{2}\right]\right\}^{1 / 2} .
$$

This equation is the model for blur-discrimination threshold as a function of reference blur and velocity for fully separable blur.

In the case of fully inseparable blurs, we treat the sum of the static spatial and velocity-dependent components as one filter, and we get a slightly different model:

$$
\Delta B=-B+\left\{B^{2}+\left(k^{2}+2 k\right)\left[B^{2}+(s+f v)^{2}\right]\right\}^{1 / 2} .
$$

If our visual system really uses special spatial filters for moving objects, there are no particular reasons that the size of the spatial filter should be a linear function of ve- 
locity. The filter size could, for instance, increase faster than the linear relation predicts. The simplest case of this kind of behavior is a quadratic velocity dependence, which gives us the following model:

$$
\Delta B=-B+\left\{B^{2}+\left(k^{2}+2 k\right)\left[B^{2}+\left(s+f v+g v^{2}\right)^{2}\right]\right\}^{1 / 2} .
$$

Cameralike blur can produce only a linear velocity dependence, and so, if this quadratic model fits the data significantly better than the model with a linear velocity dependence, we can consider it a proof of the existence of special spatial filters for moving objects.

\section{FITTING THE MODELS}

We applied a standard nonlinear least-squares routine, the Levenberg-Marquart method, ${ }^{17}$ to fit our three models to the data. We obtained the maximum-likelihood estimates of the model parameters by minimizing the $\chi^{2}$ merit function, which was defined as follows:

$$
\chi^{2}(s, f, k)=\sum_{i=1}^{N}\left[\frac{\Delta B_{i}-\Delta B(B, v ; s, f, k)}{\sigma_{i}}\right]^{2},
$$

where $\Delta B(B, v ; s, f, k)$ is the model evaluation at reference blur $B$, velocity $v$, and parameter set $\{s, f, k\}$ and $\sigma_{i}$ is the standard error for the measured value $\Delta B_{i}$. In addition to model parameters, our fitting procedure provided error estimates for the parameters and a statistical measure of goodness of fit. The probability that the true error of each parameter is less than the given estimate is $68 \%$, assuming that the errors are normally distributed. The goodness of fit of a $\chi^{2}$ fit is a function of the minimized $\chi^{2}$ value and the degrees of freedom of the fit. The quantitative absolute value of the goodness of fit is the probability $Q$ that the $\chi^{2}$ value obtained would result from chance fluctuations of the data. Both the $\chi^{2}$ value and the probability $Q$ depend on the estimates of the measurement errors. If the probability $Q$ is very small, then the discrepancies from the model are unlikely to be chance fluctuations: either the model is wrong or the measurement errors are larger than estimated. However, because of the fairly common situation of a nonnormal distribution of measurement errors, models with $Q$ values as low as 0.001 are often deemed acceptable. ${ }^{17}$

\section{RESULTS FROM THE FITTING}

With all our models and measurement data, the method was found to converge in a few iterations to the best-fit parameter set. This happened even when the initial guess values for the parameters were far from the best-fit set, indicating that the minima found by the method are the true global ones. In all cases this was confirmed by graphical inspection of the merit function, which also ruled out the existence of any other significant local minimum; i.e., the merit functions were found to be unimodal, with a single minimum.

Figure 4 shows the fit of the fully inseparable blur model with a linear velocity dependence superimposed upon the original data. The model fits the data well for each ob- server, as can also be seen from the $Q$ values in Table 1 . The number of independent data points is 24 for each observer, and there are 3 parameters in the model; thus the number of degrees of freedom is 21 . A rule of thumb in chi-square fitting states that a typical value of $\chi^{2}$ for a moderately good fit is approximately equal to the degrees of freedom of the fit, and for observers RO and AP the $\chi^{2}$ values are not far from that. The fits for observer MS are not so good, but the $Q$ values are still several orders of magnitude higher than 0.001 .

The fit for the fully separable blur model is only slightly worse than the fit for the fully inseparable blur model. It is a mathematical certainty that, having one more parameter, the fully inseparable blur model with a quadratic velocity dependence will provide a fit with a smaller $\chi^{2}$ value than the model with a linear velocity dependence. The difference in our data, however, is minimal and has no statistical significance. In fact the $Q$ values for observers $\mathrm{RO}$ and AP show a better fit in the linear than in the quadratic case. For observers RO and AP the value for the second-order parameter $g$ is less than half of its standard error, showing also at a glance that $g$ does not significantly
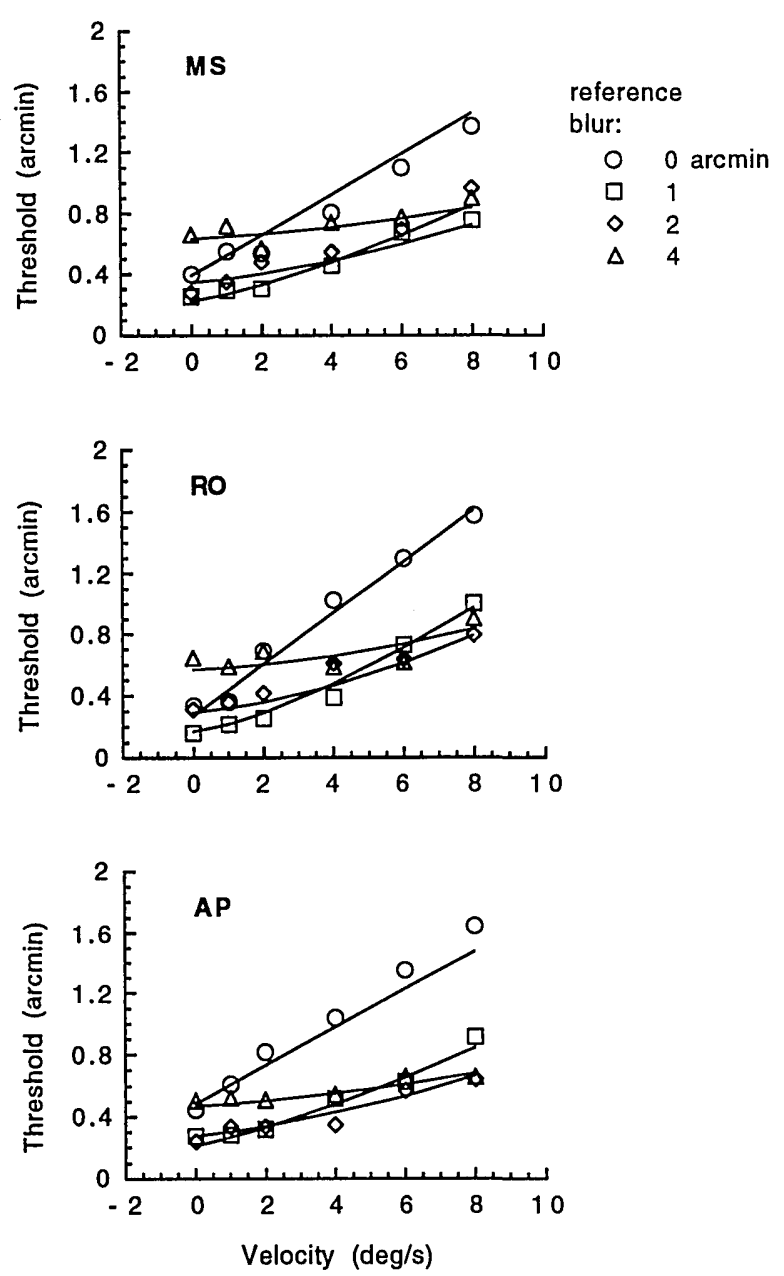

Fig. 4. Blur-model fit superimposed upon the original blurdiscrimination threshold data from Fig. 2. The model employed here assumes that the static and the velocity-dependent components of the effective spatial filter are fully inseparable and that the velocity dependence is linear. The fit provided by the fully separable blur model is almost as good as this fit. 
Table 1. Modeling of Blur-Discrimination Thresholds: Best-Fit Parameters, Their Standard Errors, and $\chi^{2}$ and $Q$ Values for Three Different Models, for Three Observers

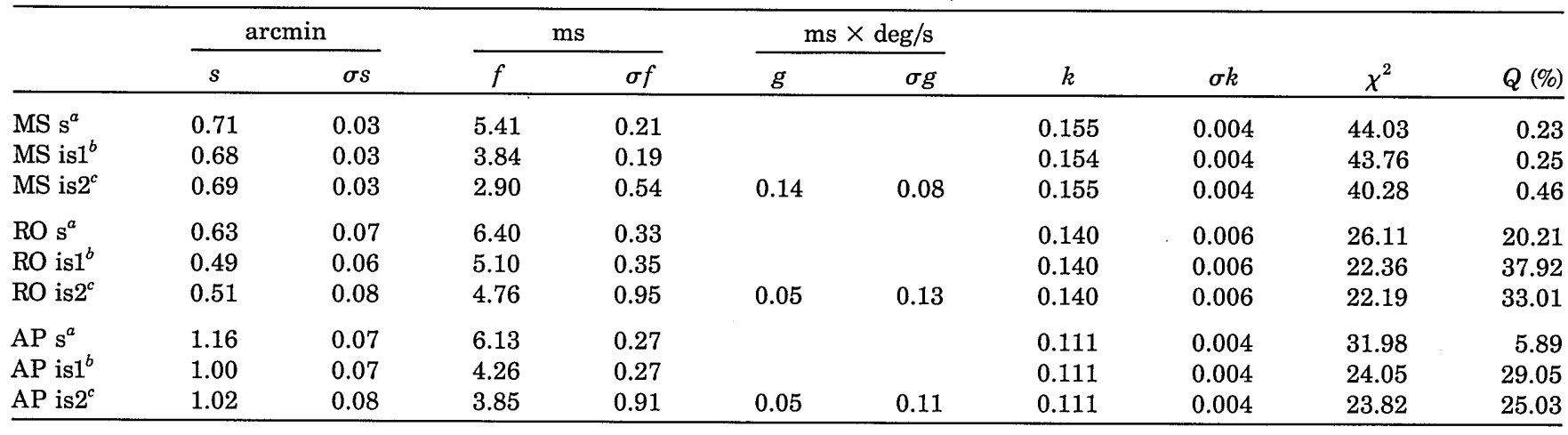

${ }^{a}$ Fully separable blur.

${ }^{b}$ Fully inseparable blur with a linear velocity dependence.

${ }^{c}$ Fully inseparable blur with a quadratic velocity dependence.

differ from zero. The same is true for observer MS, although not equally self-evidently. Thus the velocity dependence of the effective blur is linear, and we can discard the model that has a quadratic velocity dependence.

The value of the parameter $s$ provides the space constant of the effective static spatial filter. This filter corresponds to the compound effect of all the optical and neural static spatial blurring factors. For observers MS and RO, $s$ is in the range of $0.5-0.7$ arcmin. The same filter of observer AP is considerably larger, having a space constant of $\sim 1.1$ arcmin.

Assuming cameralike motion blur, the parameter $f$ is the standard deviation of the temporal weighting function, or the temporal impulse response of the system. This is why in Table 1 the parameter $f$ is expressed in milliseconds. For all the observers, $f$ is in the range of 4-6 ms, having an approximate mean of $\sim 5 \mathrm{~ms}$.

If the visual system uses special spatial filters in the perception of moving objects, then the parameter $f$ indicates simply how the size of the spatial filter depends on the velocity. In this case milliseconds are not the best temporal units for showing the strength of the dependence. When expressed in units of reference blur and velocity, the value of parameter $f$ varies between 0.23 and $0.38 \operatorname{arcmin} \times \mathrm{s} / \mathrm{deg}$. If the size of the effective static spatial filter is $\sim 0.6$ arcmin, as it is for observers MS and $\mathrm{RO}$, the effective static spatial blur is larger than the equivalent spatial blur produced by motion (i.e., $v f$ ) up to a velocity of $\sim 2 \mathrm{deg} / \mathrm{s}$. For observer AP with a larger static spatial-filter size, the corresponding limit goes to $\sim 3 \mathrm{deg} / \mathrm{s}$. Thus there is a limiting velocity of $2-3 \mathrm{deg} / \mathrm{s}$, above which the effects of the velocity-dependent part of blurring dominate. This holds both for the cameralike motion blur and for the case in which the size of the filter is assumed to increase with velocity.

The value of the parameter $k$ provides the Weber fraction for the comparison of internal representations of blur. For each observer the value of $k$ is almost the same for every model. This results from the fact that in every model $k$ is actually a similar multiplier of the term that contains all the blurring factors. For observers MS and $\mathrm{RO}$, the value of $k$ is $\sim 0.15$. Observer AP has a $k$ value of 0.11 , indicating that he has a slightly better accuracy in blur comparison than the other observers.

\section{DISCUSSION OF THE RESULTS}

\section{Velocity Dependence of the Effective Blur}

Our modeling shows that the velocity dependence of the effective blur produced by motion is linear. This is an important finding, as it shows that there does not necessarily have to be any type of motion blur other than cameralike blur to produce our results. In fact, the strict linearity of the relationship makes it tempting to believe that cameralike blur is at least the dominant component of motion blur. Since the task in our experiment was one dimensional, in the sense that we were studying the blur along the direction of motion, our data and models cannot discriminate between cameralike blur and the case in which the motion-filter size is a linear function of velocity. We tried to solve the problem by measuring discrimination thresholds for Gaussian-blurred edges that had motion trajectories perpendicular to the edge profile. The results for observer AP showed that, in this case, velocity had no discernible effect on the thresholds over the velocity range of $0-8 \mathrm{deg} / \mathrm{s}$. This result can be regarded as evidence against any isotropic motion filter, since with this kind of filter the blurring effects should be similar in all directions of motion. We can, however, present another possible explanation of why perpendicular motion did not affect the thresholds. The band that we used was exactly the same as the one used in the main experiment, being $20 \mathrm{arcmin}$ in height. With a velocity of $8 \mathrm{deg} / \mathrm{s}$, it takes more than $40 \mathrm{~ms}$ for the band to sweep over a horizontal line on the retina; i.e., there is a horizontal stripe on the retina that keeps the same luminance profile for more than $40 \mathrm{~ms}$ and even longer with lower velocities. If the blur-producing motion-integration time is less than $40 \mathrm{~ms}$, the visual system can use the same filters as for stationary objects, thus also producing the same amount of blur.

\section{Effects of the Reference Blur}

Our models predict that, as a function of reference blur, blur-discrimination thresholds approach asymptotically a Weber's-law relation. This is different from the results of Watt and Morgan, ${ }^{9}$ who found that, beyond the optimum level, thresholds rise corresponding approximately to a power law with an exponent of 1.5. We believe that the explanation of the difference may be that Watt and Morgan 
used a smaller height of ribbon (12 arcmin), which was too small for complete vertical integration of the largest blurs that they used. There is some evidence from gratingvisibility ${ }^{18,19}$ and spatial-discrimination ${ }^{20}$ experiments for this kind of integration. To test this interpretation, observer AP measured blur-discrimination thresholds for an 8-arcmin reference blur that had either a 20 - or a 40 arcmin band height. The doubling of the band height was done by halving the viewing distance; because of technical difficulties we could not increase the physical height of the band. The results confirmed that the band height affects thresholds. With the 40-arcmin band, thresholds were close to the values predicted by the models with parameters obtained from the results for the smaller reference blurs; but with the 20-arcmin band, thresholds were elevated. Most of the studies for spatial-dilation thresholds have found, as did our study, a power law with an exponent of 1.0 (i.e., Weber's law). For example, Campbell et al. ${ }^{21}$ found that spatial-frequency difference thresholds are a fixed proportion of the criterion frequency. Levi and Klein ${ }^{22}$ reported that spatial-interval discrimination thresholds are directly proportional to the separation of the lines.

\section{Effective Static Spatial Filter}

Our estimate for the space constant of the effective static spatial filter is in the range of 0.5-0.7 arcmin. Levi and Klein $^{22}$ studied the effects of Gaussian blur on two-line resolution and found that, when the stimulus blur exceeds a certain point, thresholds are degraded. They defined this transition point as the equivalent intrinsic blur. According to their measurements, the equivalent intrinsic blur in the fovea is approximately $0.4-0.7 \mathrm{arcmin}$. This value is comparable with our estimate, which is exactly what one would expect, since the equivalent intrinsic blur is the same as the effective static spatial filter; the former just is expressed in blurring terminology and the latter in filtering terminology. The diameter of the excitatory center of a Laplacian of a Gaussian filter is $\sim 2.8$ times the space constant, and, if we assume that our effective static spatial filter has the same form, then the estimate for the diameter of the excitatory center of our filter is in the range of 1.4-2.0 arcmin. This estimate is consistent with the results of Marr et al. ${ }^{23}$; these authors suggested, from the data on two-point acuity, that the smallest receptive field for a foveal mechanism in human vision must have a central diameter of between 1 and 2 arcmin. Our effective static spatial filter is also approximately the same size as the smallest filter in the pattern-discrimination models of Nielsen et al. ${ }^{24}$ and of Watt and Morgan ${ }^{25}$ but only approximately half of the smallest filter used by Wilson and Gelb. ${ }^{26}$

\section{Weber Fraction}

The best-fit values for the Weber fraction in our data are in the range of $11-16 \%$. These values are higher than those that are normally observed in spatial-dilation studies. For example, Campbell et al. ${ }^{21}$ found a Weber fraction of $6 \%$ for sine wave period discrimination. Greenlee et $a l .^{27}$ found similar values for drifting sine wave gratings. The values for spatial interval discrimination have also been found to be constant at approximately $5 \% .^{22,28,29}$ Our results can be compared with the results of the study by Watt and Morgan. ${ }^{9}$ For the reference blurs above 4 arcmin, their data showed blur-difference thresholds in the range of 10-20\%, and, even after an approximate correction for the small band height that they used, their thresholds would be higher than $6 \%$.

The difference in Weber fractions between spatial-dilation tasks and blur-discrimination tasks suggests that the blur-discrimination process may use spatial measurements that are not so accurate as the measurements in spatialinterval discrimination. To illustrate the issue, let us assume, ${ }^{30}$ for example, that our effective spatial filter has the form of a Laplacian of a Gaussian and that the visual system uses peaks and troughs in the output of this filter as spatial primitives. In the case of a Gaussian-blurred edge, the output is biphasic, containing only one peak and one adjacent valley. Watt and Morgan ${ }^{9}$ showed that the distance between the peak and the valley encodes the extent of edge blur in a way similar to the performance of human observers. In the case of a normal spatial-interval discrimination task, i.e., two thin bars separated by more than the extent of the filter, the same filter produces an output that contains two separate peaks, both with flanking troughs, making six spatial primitives altogether. Even with equally blurred edge and bars, the peaks from the bars are much narrower; i.e., they have a much better positional accuracy than the peak or the trough from the edge. Even if the accuracies of the positions of the elementary primitives were the same, having fewer primitives (or samples) in the blur-discrimination process would mean that the process would have to require a larger difference in cue, i.e., a higher Weber fraction, to produce the same statistical significance. This example explanation was based on the use of spatial primitives, but we think that this principle of differences in positional accuracy in explaining the differences in Weber fractions would also hold for a case in which there were no implicit primitives and in which the comparison would be based on the whole filtered output.

\section{Temporal Integration}

Our estimate for the standard deviation of the temporal impulse response of the system is in the range of 4-6 ms. This is the maximum estimate based on the assumption that all the blur produced by motion is cameralike. The temporal impulse responses presented in the literature are typically biphasic, having an excitatory phase followed by a smaller inhibitory phase. ${ }^{31}$ Standard deviation is a measure of probability distributions, and it is not the best descriptor for a weighting function that contains both positive and negative parts. To compare our result with values in the literature, we assume (supported by our results from some approximate simulation tests) that our estimate reflects mainly the spread of the dominant excitatory part of the impulse response function.

The temporal extent of the impulse response depends strongly on the adaptation level: the impulse response becomes shorter when the retinal illuminance increases. This makes direct comparisons between different studies difficult. Probably the lighting conditions closest to those used in our study were those used in the study of McKee and Taylor, ${ }^{32}$ in which overhead fluorescent lighting provided a background luminance of $50 \mathrm{~cd} / \mathrm{m}^{2}$. McKee and Taylor reported their results as best-fit parameters to a model proposed by Watson. ${ }^{31}$ This model is basically a difference of two gamma functions, and similar models 
have been used successfully to fit both psychophysical ${ }^{33,34}$ and neurophysiological ${ }^{35}$ data. Using the model and the given parameter values, we calculated the standard deviations for the excitatory phases of the temporal impulse response functions. For the foveal vision of the two observers of McKee and Taylor, we obtained values of 5.8 and $6.0 \mathrm{~ms}$. With the same method we obtained values of $8.3 \mathrm{~ms}$ for the temporal impulse response of Watson ${ }^{31}$ that was based on the data of Roufs and Blommaert ${ }^{36}$ and of $9.5 \mathrm{~ms}$ for the result of Bergen and Wilson. ${ }^{34} \mathrm{Kelly}^{37}$ derived temporal impulse responses for different adapting levels, and one of those levels is close to that in our study. From Kelly's plot we estimated that the excitatory phase of this temporal impulse response has a standard deviation of $\sim 5.5 \mathrm{~ms}$. Thus our estimate of $4-6 \mathrm{~ms}$ is reasonably consistent with the values obtainable from the literature for similar lighting conditions.

Instead of assuming a single filter, we could consider the possibility that the dominant source of motion is the increase in spatial-filter size with velocity. If this were the case, then our estimate for the temporal parameter $f$ would be an overestimate for the standard deviation of the temporal impulse response, because it would also contain the component from the size increase. However, the comparison with the values from the literature presented above shows that our estimate is more likely to be a slight underestimate than an overestimate. There are three possible explanations for this. First, one could speculate that the temporal impulse response in our task is briefer than in the tasks studied in the literature. This seems unlikely. Second, some motion-deblurring mechanism could remove the blur that is in excess of that predicted from the temporal impulse response, i.e., remove the amount of blur corresponding to the increase in spatial filter size. It is hard to see any rational motive for this explanation, either. The third explanation is the simplest and the most likely one. It assumes that motion blur is cameralike and not a result of an increase in spatial-filter size with velocity.

If we assume that the temporal impulse response has the form proposed by Watson ${ }^{31}$ and that the exposure or motion-integration time corresponds mainly to the width of the excitatory phase, we obtain an estimate for the motion-integration time that is approximately in the range of $20-25 \mathrm{~ms}$. However, there is other evidence from motion-detection studies that motion detectors summate for $\sim 100 \mathrm{~ms}^{4,5}$ Thus, in close agreement with the findings of Burr, ${ }^{38}$ our estimate of motion blur corresponds to only about a fifth or a fourth of what would be expected from the summation time. If we assume that the visual system uses the same mechanism for the detection and the spatial analysis of a moving object, this difference cannot be explained without some kind of blur-free integration mechanism. In what follows we discuss the timespace model of Burr et $a l .^{5}$ and the linear shifter circuit concept of Anderson and van Essen ${ }^{6}$ in the light of our findings.

\section{GENERAL DISCUSSION}

\section{Spatiotemporal Receptive Fields}

Burr et al..$^{5}$ derived the spatiotemporal receptive fields by inverse Fourier transform of the spatiotemporal tuning functions constructed with the use of masking results. According to Burr et al., motion smear is determined by the width of the central region of the spatiotemporal receptive field and by the interaction of several motion detectors. They also stated that, in principle, definition as precise as desired may be obtained by the cooperative action of many fields of different profiles, but they did not propose any mechanism for the cooperation.

Our results are consistent with the view that the apparent spatial filter at each velocity is the result of a convolution between the static spatial filter and the spatial filter that is determined by the temporal impulse response and the velocity. In the case in which the static and the velocity-dependent components are fully separable, the space constant of the apparent spatial filter follows Eq. (1). To see whether this equation also applies to the spatiotemporal receptive fields derived by Burr et al., ${ }^{5}$ we estimated the space constants from the graphic plots that Burr et al. presented for spatiotemporal receptive fields tuned to velocities of $0,1.6,8$, and $80 \mathrm{deg} / \mathrm{s}$. We did this by measuring the width of the positive central region at $t=0$ and by assuming that this one-dimensional profile has approximately the form of a second derivative of a Gaussian. Figure 5 shows the fit of Eq. (1) to the extracted values. The fit is good, indicating that the broadening of the receptive fields with velocity may result from motion blur produced by a constant integration time. The fitted parameters, however, are different from our results. The space constant of the assumed static spatial filter is $\sim 3$ arcmin, six times the mean value that we obtained. One way to explain this difference is to assume that the blur-discrimination process uses the smallest spatial filter available, whereas the process of detecting the direction of drift of a sinusoidal grating at threshold uses a much larger effective spatial filter. The difference in the temporal parameter is more important. The estimate for the standard deviation of the temporal weighting function is $28 \mathrm{~ms}$, making the temporal integration time $\sim 100 \mathrm{~ms}$. This implies that in the psychophysical task of Burr et al. ${ }^{5}$ the whole summation time produces blur, in contrast to the task of blur discrimination.

What we are suggesting here is an alternative explanation for the spatiotemporal receptive fields derived by

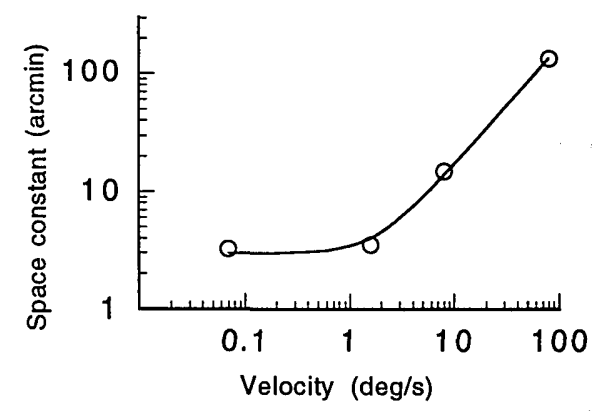

Fig. 5. Estimated space constants extracted from the graphic plots of the spatiotemporal receptive fields by Burr et al. ${ }^{5}$ (open circles) are fitted by a model that assumes that the apparent spatial filter at each velocity is a result of the convolution between the effective static spatial filter and the spatial filter determined by the temporal impulse response and the velocity. The good fit indicates that the broadening of the receptive fields with velocity can be explained by motion blur produced by a constant integration time. 
Burr et al. In this explanation the receptive fields are not the receptive fields of actual single mechanisms but instead are descriptive functions that show how motion blur, i.e., the velocity and the temporal filtering, modifies the appearance of the underlying spatial filter in spacetime coordinates.

\section{Linear Shifter Circuits}

When proposing the shifter circuits principle for blur prevention of moving objects, Anderson and van Essen ${ }^{6}$ did not make any predictions of the amounts of blur that their system would remove with different velocities. Our results show that the velocity dependence of the effective blur produced by motion is linear. Can this kind of dependence be produced by shifter circuits? In principle, the shifter circuitry produces blur if the shift control is not accurate. In blur prevention the shift control is guided by the velocity information. When the motion starts, some time passes before the shift control returns a correct velocity value, and blur is produced. The amount of blur is the product of the delay time and the velocity, and, if the delay is constant, the amount of blur depends linearly on the velocity. However, as soon as the velocity feedback signal reaches the right value, no more blur is produced. If the delay time is less than $50 \mathrm{~ms}$ and the motion-summation time is $100 \mathrm{~ms}$, then the final internal image for our 150ms presentation should be blur free. If the sum of the delay time and the summation time is more than $150 \mathrm{~ms}$, some blur is left in the final image, and the amount of this blur relates linearly to the velocity. This, however, is an oversimplification of the situation. We have not taken into account the fact that the velocity feedback signal has its own uncertainty, which also produces blur. If the error of the velocity signal is a linear function of velocity, then the blur produced by this uncertainty depends linearly on the velocity, as does also the total amount of blur. In reality, this is not necessarily the case. De Bruyn and Orban $^{39}$ measured velocity-discrimination thresholds for random-dot patterns and moving light bars. At velocities of $1,2,4$, and $8 \mathrm{deg} / \mathrm{s}$, the Weber fractions for velocity discrimination were approximately $0.12,0.09,0.065$, and 0.05 , respectively, thus nowhere near a linear relation. If these thresholds reflect the behavior of the hypothetical velocity feedback signal, as one would expect, then the amount of blur produced by the shifter circuits should not be a linear function of velocity. Since the amount of blur depends linearly on velocity, we conclude that linear shifter circuits are not the mechanism behind the blur prevention.

\section{Motion Blur versus Static Spatial Blur}

Morgan and Benton ${ }^{7}$ assumed that motion introduces spatial blur. Their explanation for blur-free percepts of moving objects was that motion blur remains undetected because the internal representation of the object is already even more degraded by static spatial blur. Our results show that this is true up to velocities of $\sim 2 \mathrm{deg} / \mathrm{s}$. Morgan and Benton argued against any motion-deblurring mechanism, but we found that the effective spatial blur produced by motion in a blur-discrimination task is much less than that predicted by a summation time of $\sim 100 \mathrm{~ms}$, indicating the existence of at least some kind of blur-free integration mechanism.

\section{Model for Temporal Integration}

The essence of both the shifter circuit and the spatiotemporal receptive-field principle is that motion blur is reduced or eliminated because the mechanisms take into account the temporal delay at which different photoreceptors have been stimulated; i.e., integration follows the path of the moving object. Some recent studies, however, suggest that blur-free integration may not be dependent on linear motion. Badcock and Wong ${ }^{40,41}$ measured separation-discrimination thresholds for a line pair and found that positional jitter of as high as 8 arcmin had little detrimental effect on performance for two lines with a separation of 6 arcmin. They produced jitter by presenting the line pair in a new randomly chosen location for $3 \mathrm{~ms}$ in every $30 \mathrm{~ms}$. They also found that performance improves with durations of up to at least $300 \mathrm{~ms}$.

We propose a model for temporal integration that is also consistent with the results reported by Badcock and Wong. ${ }^{40,41}$ In this model the integration happens in two phases. The first phase is a cameralike exposure phase governed by the temporal impulse response of the system. This phase always produces motion blur. The second phase integrates the images produced by the first phase over a time period of more than $100 \mathrm{~ms}$ in daylight. The second phase is translation invariant: it uses a mechanism that can take into account changes of object location even if the changes are random. This phase does not produce motion blur. However, whatever the method of superimposing the information of the first phase images, spatial or luminance changes between the parts of the object lead to image degradation that may be difficult to discriminate from motion blur. The second phase does not remove blur, either; in fact removing blur would be dangerous, since at that point the system could have no way of knowing what is blur in the image. Instead, the aim of this phase is to increase the signal-to-noise ratio or the statistical significance in the discrimination of the features in the image. In perceptual terms, this phase increases the perceived clarity of the image. The second phase integration in our model parallels closely the ideas of Dodwell, ${ }^{42}$ who presented a model that is based on correlation of successive time samples of retinal inputs, perceptual clarity being attained by a form of autocorrelation.

Badcock and Wong ${ }^{40,41}$ found that positional jitter did not degrade performance in a line-separation task when the line pair was updated in a random position every $30 \mathrm{~ms}$. Our model provides a simple explanation for this result: since there are no spatial displacements inside the first-phase integration time of $\sim 25 \mathrm{~ms}$, no blur is expected. However, our model predicts that, if the time interval between the presentations of the line pairs is made shorter than $\sim 25 \mathrm{~ms}$, blur will start to degrade the performance.

When we were modeling the receptive field sizes of Burr et al., ${ }^{5}$ using the assumption that the apparent widening of the fields is produced by motion blur, we found a temporal integration time of $\sim 100 \mathrm{~ms}$. In our own blurdiscrimination task, the estimate for the blur-produced temporal integration is only $\sim 25 \mathrm{~ms}$. Our two-phase temporal integration model can give a qualitative explanation of this difference. The object in the blur-discrimination task, i.e., the moving bar, remains constant, and thus blur is introduced only in the first integration phase. Burr 
et $a l .,{ }^{5}$ on the other hand, used drifting sinusoidal gratings seen through a stationary window. This kind of grating is not a constant moving object. The phase shift between the grating and the window changes continuously, and if the second-phase integration mechanism uses, for instance, the center of luminance in the window to superimpose the first phase images, the images are not superimposed in phase. Thus the grating is also blurred in the second-phase integration.

Recently, Watamaniuk ${ }^{43}$ presented results that at first glance may seem contradictory to those produced by our model. Using dynamic dot cinematograms, he showed that points moving in constant directions, or fixed trajectories, show less persistence than points moving with the same temporal and spatial step sizes but taking random walks. As a measure of persistence he used the apparent number of points in the display. Our model can qualitatively explain the difference in persistence between the fixed-trajectory and random-walk conditions. Assume, for instance, that the frame rate of the cinematogram display is such that each moving dot is represented as three dots corresponding to three consecutive positions of the dot in the first-phase image of our model. If the dot moves in a constant direction, only a translational shift is needed to superimpose two consecutive first-phase images, and the translation-invariant second phase can do this without more points being added to the integral image. However, if the dot moves along a random-walk trajectory, no translational or rotational, shift can completely superimpose two consecutive first-phase images, and there will be more than three points in the integral image. This explanation is oversimplified in that it does not take into account the temporal impulse response that changes the relative intensities of the three apparent dots between successive first-phase images and thus affects the superimposition, but nevertheless it demonstrates the general principle. The explanation is also supported by Watamaniuk's results that showed the difference in persistence between the fixed-trajectory and the randomwalk conditions to decrease when the frame rate was lowered from 60 to 40 and further to $20 \mathrm{~Hz}$. Our proposal for temporal integration is also in agreement with Watamaniuk's finding that the reduction of visible persistence with fixed-trajectory motion is governed solely by step size, i.e., displacement, and not by speed.

Our model for temporal integration is not a motiondeblurring model, since it does not take into account the temporal delay at which different photoreceptors have been stimulated, nor does the integration follow the path of the moving object. To put it simply, our model is not motion tuned. It explains the resistance of the visual system to positional noise when the random shifts of the target are introduced with time intervals longer than $\sim 25 \mathrm{~ms}$ and within a limited spatial window, although our experiment does not allow us to make any predictions about the size of this window. The essence of our model and of our interpretations of the findings in this study is that the form of a moving object is determined by use of the same or similarly sized spatial filter or filters as for stationary objects up to velocities of at least $8 \mathrm{deg} / \mathrm{s}$. In neurobiological terms, the high spatial resolution found in our blur-discrimination experiment is consistent with the idea implicit, for instance, in the paper of Livingstone and
Hubel $^{44}$ that the parvocellular-interblob stream is involved in high-resolution form perception, even with moving targets.

\section{ACKNOWLEDGMENTS}

Ari Pääkkönen was supported by a research grant from the Medical Research Council of the Academy of Finland and by a scholarship from the Faculty of Medicine of the University of Edinburgh.

*Present address, Department of Clinical Neurophysiology, Kuopio University Hospital, SF-70210 Kuopio, Finland.

\section{REFERENCES}

1. G. Westheimer and S. P. McKee, "Visual acuity in the presence of retinal image motion," J. Opt. Soc. Am. 65, 847-850 (1975).

2. G. Westheimer and S. P. McKee, "Stereoscopic acuity for moving retinal images," J. Opt. Soc. Am. 68, 450-455 (1978).

3. H. B. Barlow, "Temporal and spatial summation in human vision at different background intensities," J. Physiol. 141, 337-350 (1958).

4. D. C. Burr, "Temporal summation of moving images by the human visual system," Proc. R. Soc. London Ser. B 211, 321339 (1981).

5. D. C. Burr, J. Ross, and M. C. Morrone, "Seeing objects in motion," Proc. R. Soc. London Ser. B 227, 249-265 (1986).

6. C. H. Anderson and D. C. van Essen, "Shifter circuits: a computational strategy for dynamic aspects of visual processing," Proc. Natl. Acad. Sci. (USA) 84, 6297-6301 (1987).

7. M. J. Morgan and S. Benton, "Motion-deblurring in human vision," Nature (London) 340, 385-386 (1989).

8. M. J. Morgan, "Hyperacuity," in Spatial Vision, D. Regan, ed. (Macmillan, London, 1991).

9. R. J. Watt and M. J. Morgan, "The recognition and representation of edge blur: evidence for spatial primitives in human vision," Vision Res. 23, 1464-1477 (1983).

10. R. J. Watt and D. P. Andrews, "APE: adaptive probit estimation of psychometric functions," Curr. Psychol. Rev. 1, 205214 (1981).

11. D. J. Finney, Probit Analysis (Cambridge U. Press, Cambridge, 1971).

12. H. B. Barlow and W. R. Levick, "Three factors limiting the reliable detection of light by retinal ganglion cells in the cat," J. Physiol. 200, 1-24 (1969).

13. H. B. Barlow and W. R. Levick, "Changes in the maintained discharge with adaptation level in the cat retina," J. Physiol. 202, 699-718 (1969).

14. A. M. Derrington and P. Lennie, "Spatial and temporal contrast sensitivities of neurons in the lateral geniculate nucleus of macaque," J. Physiol. 357, 219-240 (1984).

15. D. Fleet, P. E. Hallett, and A. D. Jepson, "Spatiotemporal inseparability in early visual processing," Biol. Cybern. 52, 153-164 (1985).

16. R. J. Watt, Visual Processing (Erlbaum, Hove, East Sussex, UK, 1988).

17. I. Press and H. William, Numerical Recipes in C (Cambridge U. Press, Cambridge, 1988).

18. E. R. Howell and R. F. Hess, "The functional area for summation to threshold for sinusoidal gratings," Vision Res. 18, 369-374 (1978).

19. J. G. Robson and N. Graham, "Probability summation and regional variation in contrast sensitivity across the visual field," Vision Res. 21, 409-418 (1981).

20. D. M. Levi and S. A. Klein, "Sampling in spatial vision," Nature (London) 320, 360-362 (1986).

21. F. W. Campbell, J. Nachmias, and J. Jukes, "Spatial-frequency discrimination in human vision," J. Opt. Soc. Am. 60, 555559 (1970).

22. D. M. Levi and S. A. Klein, "Equivalent intrinsic blur in spatial vision," Vision Res. 30, 1971-1993 (1990). 
23. D. Marr, T. Poggio, and E. Hildreth, "Smallest channel in early human vision," J. Opt. Soc. Am. 70, 868-871 (1980).

24. K. R. K. Nielsen, A. B. Watson, and A. J. Ahumada, "Application of a computable model of human spatial vision to phase discrimination," J. Opt. Soc. Am. A 2, 1600-1606 (1985).

25. R. J. Watt and M. J. Morgan, "A theory of the primitive spatial code in human vision," Vision Res. 25, 1661-1674 (1985).

26. H. R. Wilson and D. J. Gelb, "Modified line-element theory for spatial-frequency and width discrimination," J. Opt. Soc. Am. A 1, 124-131 (1984).

27. M. W. Greenlee, J. Gerling, and S. Waltenspiel, "Spatialfrequency discrimination of drifting gratings," Vision Res. 30, 1331-1339 (1990).

28. G. Westheimer and S. P. McKee, "Integration regions for visual hyperacuity," Vision Res. 17, 89-93 (1977).

29. R. J. Watt, "Towards a general theory of the visual acuities for shape and spatial arrangement," Vision Res. 24, 13771386 (1984).

30. R. J. Watt and M. J. Morgan, "Spatial filters and the localization of luminance changes in human vision," Vision Res. 24, 1387-1397 (1984).

31. A. B. Watson, "Derivation of the impulse response: comments on the method of Roufs and Blommaert," Vision Res. 22, 1335-1337 (1982).

32. S. P. McKee and D. G. Taylor, "Discrimination of time: comparison of foveal and peripheral sensitivity," J. Opt. Soc. Am. A 1, 620-627 (1984).

33. J. Z. Levinson, "Flicker fusion phenomena," Science 160, 2128 (1968).

34. J. R. Bergen and H. R. Wilson, "Prediction of flicker sensi- tivities from temporal three-pulse data," Vision Res. 25, 577582 (1985).

35. D. A. Baylor, A. L. Hodgkin, and T. D. Lamb, "Reconstruction of the electrical responses of turtle cones to flashes and steps of light," J. Physiol. 242, 759-791 (1974).

36. J. A. J. Roufs and F. J. J. Blommaert, "Temporal impulse and step responses of the human eye obtained psychophysically by means of a drift-correcting perturbation technique," Vision Res. 21, 1203-1221 (1981).

37. D. H. Kelly, "Theory of flicker and transient responses. I. Uniform fields," J. Opt. Soc. Am. 61, 537-546 (1971).

38. D. Burr, "Motion smear," Nature (London) 284, 164-165 (1980).

39. B. De Bruyn and G. A. Orban, "Human velocity and direction discrimination measured with random dot patterns," Vision Res. 28, 1323-1335 (1988).

40. D. R. Badcock and T. L. Wong, "Resistance to positional noise in human vision," Nature (London) 343, 554-555 (1990).

41. D. R. Badcock and T. L. Wong, "The sensitivity of separation discrimination to spatiotemporal jitter," Vision Res. 30, 1555-1560 (1990).

42. P. C. Dodwell, "On perceptual clarity," Psychol. Rev. 78, 275289 (1971).

43. S. N. J. Watamaniuk, "Visual persistence is reduced by fixedtrajectory motion but not by random motion," Perception 21, 791-802 (1992).

44. M. S. Livingstone and D. H. Hubel, "Psychophysical evidence for separate channels for the perception of form, color, movement and depth," J. Neurosci. 7, 3416-3468 (1987). 